\title{
SIGN COMPATIBLE EXPRESSIONS FOR MINORS OF THE MATRIX $I-A$
}

BY

D. J. HARTFIEL

\begin{abstract}
Let $A=\left(a_{i j}\right)$ be an $n \times n$ nonnegative matrix having row sums less than or equal to one. This paper shows that the $i j$ th minor of $I-A$ can be expressed as

$$
(-1)^{i+j} \sum \Pi r_{k} a_{p q}
$$

where

$$
r_{k}=1-\sum_{s=1}^{n} a_{k s}
$$

and each $I I r_{k} a_{p q}$ is a product of exactly $n-1$ numbers taken from $r_{k}, a_{p q}$ for $k, p, q=1, \ldots, n$. This theorem is then used to obtain perturbation results concerning the matrix $I-A$.
\end{abstract}

Perturbation results in matrix theory are concerned with estimating the error in matrix computations. This paper provides perturbation results for the matrix $I-A$ where $A=\left(a_{i j}\right)$ is nonnegative having row sums less than or equal to one. The method by which these perturbation results are achieved is a variant of that given by Sengupta [2] in his work on comparing stochastic eigenvectors of two irreducible stochastic matrices. The method, as we apply it, first gives expressions for the minors of $I-A$, in terms of the entries of $A$, and then uses these expressions to produce useful perturbation results for this matrix.

The theorem of the paper produces expressions for the minors of $I-A$.

THEOREM. Let $A$ be an $n \times n$ nonnegative matrix having largest row sum less than or equal to one. Then

$$
\left|(I-A)_{i j}\right|=(-1)^{i+j} \sum \prod r_{k} a_{p q}
$$

where

$$
r_{k}=1-\sum_{s=1}^{n} a_{k s}
$$

and each $\Pi r_{k} a_{p q}$ is a product of exactly $n-1$ numbers taken from $r_{k}, a_{p q}$ for $k, p, q=1, \ldots, n$.

Received by the editors January 16, 1978 and, in revised form, December 12, 1978.

AMS (MOS) subject classifications (1970). Primary 15A15; Secondary 15A09, $15 A 42$.

(c) 1979 American Mathematical Society 0002-9939/79/0000-0551/\$02.25 
Proof. The method of proof is induction on $n$. The case $n=2$ can be proved by checking all choices for $i$ and $j$. Thus, suppose the theorem is true for all $n \times n$ matrices $A$, satisfying the hypothesis, where $n<n_{1}$. Now let $A$ be an $n \times n$ matrix, satisfying the hypothesis, where $n=n_{1}$. The argument is divided into two cases. The first case to be considered is when $i \neq j$. Here we will assume $i<j$ as the case $i>j$ is argued similarly.

For this case, we first define an $n \times n$ matrix $E_{p q}=\left(e_{r s}\right)$ where

$$
e_{r s}= \begin{cases}1, & \text { if } r=s \text { and } r \neq p, r \neq q, \\ 1, & \text { if } r=p \text { and } s=q, \\ 1, & \text { if } r=q \text { and } s=p, \\ 0, & \text { otherwise. }\end{cases}
$$

Let $P=E_{12} E_{23} \cdots E_{i-1, i}$, a permutation matrix, and set $P(I-A) P^{t}=I-$ $P A P^{t}=I-B$. Then

$$
\left|(I-A)_{i j}\right|=(-1)^{i-1}\left|(I-B)_{1 j}\right| .
$$

Hence we need only prove the result for $i=1$ and $j>1$.

For this then we expand $\left|(I-A)_{1 j}\right|$ about the lst column achieving that

$$
\left|(I-A)_{1 j}\right|=\sum_{s>1}(-1)^{s-1+1}\left(-a_{s 1}\right)\left|\left[(I-A)_{1 j}\right]_{s 1}\right|
$$

where $\left[(I-A)_{1 j}\right]_{s 1}$ denotes the matrix obtained from $(I-A)$ by deleting rows $1, s$ and columns $j, 1$.

Now noting that $\left[(I-A)_{1 j}\right]_{s 1}=\left[(\dot{I}-A)_{11}\right]_{s j}$ and applying the induction hypothesis yields that

$$
\begin{aligned}
\left|(I-A)_{1 j}\right| & =\sum_{s>1}(-1)^{s+1} a_{s 1}\left|\left[(I-A)_{11}\right]_{s j}\right| \\
& =\sum_{s>1}(-1)^{s+1} a_{s 1}\left((-1)^{s+j} \sum \prod\left(r_{k}+a_{k 1}\right) a_{p q}\right) \\
& =(-1)^{1+j} \sum \prod r_{k} a_{p q}
\end{aligned}
$$

where each $\Pi r_{k} a_{p q}$ is a product of exactly $n-1$ numbers taken from $r_{k}, a_{p q}$ for $k, p, q=1, \ldots, n$.

For the case $i=j$, we assume without loss of generality that $i=j=1$. Write

$$
(I-A)_{11}=\left(\begin{array}{cccc}
r_{2}+\sum_{s \neq 2} a_{2 s} & -a_{23} & \ldots & -a_{2 n} \\
-a_{32} & r_{3}+\sum_{s \neq 3} a_{3 s} & \ldots & -a_{3 n} \\
\ldots \ldots \ldots \ldots & \ldots \ldots \ldots & \ldots \ldots \\
-a_{n 2} & -a_{n 3} & \ldots & r_{n}+\sum_{s \neq n} a_{n s}
\end{array}\right)
$$


Adding columns two through $n-1$ to column one yields

$$
B=\left(\begin{array}{cccc}
r_{2}+a_{21} & -a_{23} & \ldots & -a_{2 n} \\
r_{3}+a_{31} & r_{3}+\sum_{s \neq 3} a_{3 s} & \ldots & -a_{3 n} \\
\ldots \ldots & \ldots & \ldots & \ldots \\
r_{n}+a_{n 1} & -a_{n 3} & \ldots & r_{n}+\sum_{s \neq n} a_{n s}
\end{array}\right) .
$$

Expanding the determinant about the first column of $B$ and noting that $B_{s 1}=\left[(I-A)_{11}\right]_{s+1,2}$ yields, by the induction hypothesis, that

$$
\begin{aligned}
\left|(I-A)_{11}\right| & =\sum_{s=1}^{n-1}(-1)^{s+1}\left(r_{s+1}+a_{s+1,1}\right)\left|\left[(I-A)_{11}\right]_{s+1,2}\right| \\
& =\sum_{s=1}^{n-1}(-1)^{s+1}\left(r_{s+1}+a_{s+1,1}\right)\left((-1)^{s+1}\left(\sum \prod\left(r_{k}+a_{k 1}\right) a_{p q}\right)\right. \\
& =\sum \prod r_{k} a_{p q}
\end{aligned}
$$

where each $\Pi r_{k} a_{p q}$ is a product of exactly $n-1$ numbers taken from $r_{k}, a_{p q}$ for $k, p, q=1, \ldots, n$.

This theorem is now applied to yield our first perturbation result. This result estimates the error in computing $(I-A)^{-1}$, when it exists.

COROllary 1. Let $A$ and $\hat{A}$ be $n \times n$ nonnegative matrices with row sums less than or equal to one and having spectral radius less than 1 . Set $B=(I-$ $A)^{-1}$ and $\hat{B}=(I-\hat{A})^{-1}$. If

(1) $a_{i j}<\hat{\theta} \hat{a}_{i j}, r_{k}<\hat{\theta} \hat{r}_{k}$ and

(2) $\hat{a}_{i j}<\theta a_{i j}, \hat{r}_{k}<\theta r_{k}$

then, for all $b_{i j} \neq 0,\left(\hat{b}_{i j}-b_{i j}\right) / b_{i j}<\theta^{n-1} \hat{\theta}^{n}-1$.

Proof. Note that

$$
b_{i j}=\frac{(-1)^{i+j}\left|(I-A)_{j i}\right|}{|(I-A)|} \quad \text { and } \quad \hat{b}_{i j}=\frac{(-1)^{i+j}\left|(I-\hat{A})_{j i}\right|}{|(I-\hat{A})|}
$$

Thus, if $b_{i j} \neq 0$, application of the theorem yields that

$$
\frac{\hat{b}_{i j}}{b_{i j}}=\frac{\left|(I-\hat{A})_{j i}\right||I-A|}{\left|(I-A)_{j i}\right||I-\hat{A}|}<\frac{\theta^{n-1}\left|(I-A)_{j i}\right| \hat{\theta}^{n}|I-\hat{A}|}{\left|(I-A)_{j i}\right||I-\hat{A}|}<\theta^{n-1} \hat{\theta}^{n} .
$$

Hence,

$$
\frac{\hat{b}_{i j}-b_{i j}}{b_{i j}} \leqslant \theta^{n-1} \hat{\theta}^{n}-1
$$

A second perturbation result estimates the error in solving Leontif's open economic model. 
COROLlary 2. Let $A$ and $\hat{A}$ be $n \times n$ nonnegative matrices having largest row sums less than or equal to one and having spectral radii less than one. Let $b$ and $\hat{b}$ be $1 \times n$ nonnegative vectors with $x(I-A)=b$ and $\hat{x}(I-\hat{A})=\hat{b}$. If

(1) $a_{i j}<\hat{\theta} \hat{a}_{i j}, r_{k}(A)<\hat{\theta} r_{k}(\hat{A}), b_{i}<\hat{\theta} \hat{b}_{i}$ and

(2) $\hat{a}_{i j}<\theta a_{i j}, r_{k}(\hat{A})<\theta r_{k}(A), \hat{b}_{i}<\theta b_{i}$

then, for all $x_{i} \neq 0,\left(\hat{x}_{i}-x_{i}\right) / x_{i}<(\theta \hat{\theta})^{n}-1$.

Proof. Note first that $x=(I-A)^{-1} b$ and $\hat{x}=(I-\hat{A})^{-1} \hat{b}$. Then, if $x_{i} \neq 0$, application of the theorem yields that

$$
\frac{\hat{x}_{i}}{x_{i}}=\frac{\Sigma_{r}(-1)^{i+r}\left|(I-\hat{A})_{r i}\right| \hat{b}_{r}|I-A|}{|I-\hat{A}| \Sigma_{r}(-1)^{i+r}\left|(I-A)_{r i}\right| b_{r}}<(\theta \hat{\theta})^{n} .
$$

Hence

$$
\frac{\hat{x}_{i}-x_{i}}{x_{i}}<(\theta \hat{\theta})^{n}-1
$$

The last perturbation result estimates the error in computing stochastic eigenvectors for stochastic matrices.

Corollary 3. Let $A$ and $\hat{A}$ be $n \times n$ irreducible stochastic matrices. Suppose $\alpha$ and $\hat{\alpha}$ are stochastic eigenvectors, belonging to one, for $A$ and $\hat{A}$ respectively. If (1) $a_{i j}<\hat{\theta} \hat{a}_{i j}$ and (2) $\hat{a}_{i j}<\theta a_{i j}$ then $\left(\hat{\alpha}_{i}-\alpha_{i}\right) / \alpha_{i}<(\theta \hat{\theta})^{n-1}-1$.

Proof. First note that the Perron-Frobenius theory [1] gives that if $\alpha$ and $\hat{\alpha}$ are stochastic eigenvectors, belonging to one, for $A$ and $\hat{A}$ respectively, then $\alpha$ and $\hat{\alpha}$ are the unique solutions to

$$
\alpha(I-A)=0 \text { with } \sum \alpha_{i}=1 \text { and } \hat{\alpha}(I-\hat{A})=0 \text { with } \sum \hat{\alpha}_{i}=1 .
$$

Further, by the Perron-Frobenius theory, $\operatorname{rank}(I-A)=\operatorname{rank}(I-\hat{A})=n$ -1 with the first $n-1$ columns of both $I-A$ and $I-\hat{A}$ being linearly independent. Hence, the above equations are equivalent to

$$
\alpha\left[(I-A)_{n} e\right]=e_{n} \text { and } \hat{\alpha}\left[(I-\hat{A})_{n} e\right]=e_{n}
$$

where $(I-A)_{n}$ and $(I-\hat{A})_{n}$ are obtained by deleting the $n$th column of $(I-A)$ and $(I-\hat{A})$ respectively. Further $e_{i}$ is the $(0,1)$-vector having its only nonzero entry in the $i$ th position and $e=e_{1}+\cdots+e_{n}$. Now, by Cramer's rule

$$
\alpha_{i}=(-1)^{i+n} \operatorname{det}\left(\left[(I-A)_{n} e\right]_{i n}\right) / \operatorname{det}\left[(I-A)_{n} e\right]
$$

and

$$
\hat{\alpha}_{i}=(-1)^{i+n} \operatorname{det}\left(\left[(I-\hat{A})_{n} e\right]_{i n}\right) / \operatorname{det}\left[(I-\hat{A})_{n} e\right] .
$$

Thus

$$
\frac{\hat{\alpha}_{i}}{\alpha_{i}}=\frac{\operatorname{det}\left(\left[(I-\hat{A})_{n} e\right]_{i n}\right) \operatorname{det}\left[(I-A)_{n} e\right]}{\operatorname{det}\left(\left[(I-A)_{n} e\right]_{i n}\right) \operatorname{det}\left[(I-\hat{A})_{n} e\right]} .
$$


Noting that $\operatorname{det}\left[(I-\hat{A})_{n} e\right]_{\text {in }}=\operatorname{det}\left[(I-\hat{A})_{i n}\right]$ and $\operatorname{det}\left[(I-A)_{n} e\right]_{\text {in }}=\operatorname{det}[(I$ $\left.-A)_{i n}\right]$ and expanding $\operatorname{det}\left[(I-A)_{n} e\right]$ and $\operatorname{det}\left[(I-\hat{A})_{n} e\right]$ about the last column yields, by applying the theorem, that

$$
\frac{\hat{\alpha}_{i}}{\alpha_{i}}<\frac{\left(\theta^{n-1} \sum \prod r_{k} a_{p q}\right)\left(\hat{\theta}^{n-1} \sum \prod \hat{r}_{k} \hat{a}_{p q}\right)}{\left(\sum \prod r_{k} a_{p q}\right)\left(\sum \prod \hat{r}_{k} \hat{a}_{p q}\right)}<(\theta \hat{\theta})^{n-1} .
$$

Hence,

$$
\frac{\hat{\alpha}_{i}-\alpha_{i}}{\alpha_{i}}<(\theta \hat{\theta})^{n-1}-1
$$

\section{REFERENCES}

1. F. R. Gantmacher, The theory of matrices. Vol. 2, Chelsea, New York, 1960.

2. Sailes Kumar Sengupta, Comparison of eigenvectors of irreducible stochastic matrices, Linear Algebra and Appl. 12 (1975), 101-110.

Department of Mathematics, Texas AdM University, College Station, Texas 77843 